\title{
“El día que me muera me vas a escuchar": de la identidad y la violencia en el contexto del fútbol en Colombia
}

\author{
"The day I die you will listen to me": identity and violence in the context of \\ Colombian soccer \\ “O dia em que eu morrer, você vai me ouvir": da identidade e a violência no \\ contexto do futebol na Colômbia
}

John Alexander Castro-Lozano*

https://orcid.org/0000-000 I-8397-7690 Universidad Distrital Francisco José de Caldas, Bogotá, Colombia

- Fecha de recepción: 2019-II-26

- Fecha concepto de evaluación: 2019-1I-29

- Fecha de aprobación: 2019-11-30 http://dx.doi.org/I0.22335/rlct.vI2il.I I I I
Para citar este artículo / To reference this article / Para citar este artigo: CastroLozano, J.A. (2020). "El día que me muera me vas a escuchar": de la identidad y la violencia en el contexto del fútbol en Colombia. Revista Logos Ciencia \& Tecnología, I2(I), I75- I87. http:// dx.doi.org//0.22335/rlct.vI2il.IIII

\begin{abstract}
RESUMEN
Los hinchas que integran las barras bravas han dejado a un lado la pasividad del espectador y participan en enfrentamientos. Este tipo de conductas son entendidas como manifestaciones agresivas, conflictivas y violentas, actuaciones que han logrado la atención de autoridades oficiales que buscan prevenir este tipo de comportamientos, en especial en los escenarios deportivos. Sin embargo, estas actuaciones son identificadas por los hinchas que forman las barras bravas como la manifestación del "aguante". De esta manera, el artículo propone entender las formas de constitución de la identidad y las rivalidades en el contexto del fútbol. Igualmente, comprender e interpretar la noción-experiencia del "aguante" desde la perspectiva de los hinchas y desde el análisis socio antropológico. En este aspecto, los hinchas constituyen una identidad y además las rivalidades del fútbol son una expresión de la violencia a través del "aguante". Finalmente, la implementación de una política pública contra la violencia del fútbol no implica la desaparición o disminución de los comportamientos agresivos y conflictivos.
\end{abstract}

Palabras clave: identidad futbolística, rivalidades, violencia del fútbol, “aguante”, barras bravas 


\begin{abstract}
SUMMARY
The fans that are consider 'soccer hooligans' have put to one side the passivity of the spectators and, instead, participate in clashes. These types of behaviors are understood as aggressive, conflictive and violent manifestations, actions that have caught the attention of official authorities that seek to prevent this type of behavior, especially in sports settings. However, these actions are identified by the fans that are considered 'hooligans' as the manifestation of 'stamina'. Therefore, this article proposes to understand the forms of constitution of identity and rivalries in the context of football. Likewise, it pretends to understand and interpret the notion-experience of "endurance" from the perspective of the fans and from the socio-anthropological analysis. In this aspect, the fans constitute an identity, and what's more, rivalries in football are an expression of violence through 'stamina'. Finally, the implementation of a public policy against football violence does not imply the disappearance or reduction of aggressive and conflicting behaviors.
\end{abstract}

Keywords: football identity, rivalries, football violence, "stamina", 'soccer hooligans'

\title{
SUMÁRIO
}

Os torcedores que compõem as torcidas organizadas deixaram de lado a passividade de espectador e participam de confrontos. Esses tipos de comportamentos são entendidos como manifestações agressivas, conflituosas e violentas, ações que chamaram a atenção de autoridades oficiais que buscam impedir esse tipo de comportamento, principalmente em ambientes esportivos. No entanto, essas ações são identificadas pelos torcedores que formam as torcidas organizadas como manifestação de "resistência". Dessa forma, o artigo propõe entender as formas de constituição da identidade e rivalidades no contexto do futebol. Da mesma forma, entender e interpretar a noção-experiência de "resistência" da perspectiva dos torcedores e da análise sócio- antropológica. Nesse aspecto, os torcedores constituem uma identidade e, além disso, as rivalidades do futebol são uma expressão de violência através da "resistência". Finalmente, a implementação de uma política pública contra a violência no futebol não implica o desaparecimento ou a redução de comportamentos agressivos e conflitantes.

Palavras-chave: Identidade futebolística, rivalidades, violência no futebol, "resistência”, torcidas organizadas

En este trabajo, se utiliza la noción de "barras bravas" para hacer referencia a Grupos Organizados de Hinchas $(\mathrm{GOH})$ que promueven espectáculos festivos y se involucran en enfrentamientos. Por ese motivo, no serán utilizados los conceptos de "barristas", "barras futboleras" o "barras populares", ya que estos términos no consideran la participación en los enfrentamientos en los que se puedan involucrar los hinchas. Los $\mathrm{GOH}$ son denominados de distinta manera en Europa y en Suramérica: hooligans en Inglaterra, siders en los Países Bajos, ultras en Italia y España, torcidas organizadas en Brasil y barras bravas en Hispanoamérica. En Colombia, durante la década de 1990 se constituyeron las barras bravas y el surgimiento desembocó en la radicalización de las rivalidades futbolísticas, puesto que los insultos fueron más ofensivos y amenazan- tes, y los enfrentamientos se regularizaron antes, durante o después de los encuentros futbolísticos.

Por ejemplo, una de las primeras peleas ocurrió en Bogotá el 29 de septiembre de 1993. Después de concluido el clásico de Bogotá, Blue Rain del Club Deportivo Los Millonarios se enfrentó a Santa Fe de Bogotá del Club Independiente Santa Fe en los alrededores del estadio Nemesio Camacho "El Campín". Otra riña sucedió el 10 de octubre de 1993, los hinchas que formaban de Blue Rain pelearon contra hinchas de Nacional de Medellín en las graderías de "El Campín”, durante el partido de fútbol. Después de estos sucesos, los enfrentamientos lograron una regularidad y una visibilidad, pues se desarrollaron en el interior y el exterior de los estadios, en los barrios de 
distintas ciudades y en diferentes carreteras del país. El inicio del nuevo milenio trajo consigo un relevo generacional entre las barras bravas. Ese cambio radicalizó las rivalidades futbolísticas y los enfrentamientos se hicieron más peligrosos, pues la utilización de armas blancas' e, incluso, de fuego, se hizo usual.

De esta manera, el propósito de este artículo es comprender la constitución de la identidad y de las rivalidades futbolísticas. Además, explicar las (posibles) causas que provocan los comportamientos agresivos, conflictivos $y$ violentos entre los hinchas que forman las barras bravas, enfrentamientos entre rivales e, incluso, del mismo equipo de fútbol desde la noción-experiencia del "aguante"2. En otras palabras, exponer los motivos de los enfrentamientos $y$, en algunas ocasiones, de los asesinatos en el contexto del fútbol. En este aspecto, el artículo se organiza de la siguiente manera: primero, de los clubes y la constitución de las identidades futbolísticas; segundo, de la formación de las barras bravas y la configuración de las rivalidades del fútbol; tercero, una aproximación a la noción-experiencia del "aguante" (que dicen manifestar los hinchas que forman las barras bravas), que puede entenderse como la violencia futbolística; y cuarto, se presenta una elaboración propia en la que son referenciados distintos asesinatos relacionados con hinchas de clubes del Fútbol Profesional Colombiano (FPC); finalmente, son expuestas algunas conclusiones.

\section{De los clubes y las identidades futbolísticas}

Las prácticas deportivas modernas ingresaron a Colombia al final del siglo $X I X$ y al comienzo siglo $X X$. El deporte "estaba determinado por la acción de las élites locales, quienes a través de la institución de los clubes sociales encontraban el espacio ideal para desarrollar prácticas de distinción que las afirmaban socialmente como élites dominantes" (Jaramillo, 2018, p. 48). En Bogotá, Cali, Barranquilla, Pasto, Medellín, Santa Marta, Cúcuta y Pereira, se organizaron las primeras experiencias del fútbol en territorio nacional. En la década de 1910, el fútbol en Bogotá no se había profesionalizado, pero sí se habían constituido equipos y se realizaban algunas competencias. Benninghoff (200I) afirma que es posible organizar a los equipos de

I Navajas, cuchillos o machetes.

2 Las palabras o expresiones usadas por los hinchas, en el contexto del fútbol, son resaltadas en comillas.
Bogotá en tres grupos: "los que representaron a los clubes de la 'alta sociedad', los que fueron constituidos por las principales instituciones educativas de la ciudad, $y$, por último, los equipos formados por militares" (p. 16).

Algunos de los clubes en Bogotá en la década de 1910 fueron Polo Club, Bartolino, Club Inglés, Club Colombia, Club Bogotá, Escuela Militar, Tequendama Football Club, Santander Football Club, Club Chile, Club Liberty, City Club, Club República, Club América, Club Nariño, Bolívar,Tarqui, Ricaurte, Sporting, Stard Club, The Albion, El Rosario, Club Unión, entre otros (Santos, 2005). Desde la década de 1910 hasta la década de 1940, se organizaron competencias municipales, departamentales y regionales de fútbol aficionado. Sin embargo, el FPC inició en 1948 y, así, comenzó la configuración de los sujetos y de los grupos sociales en el contexto del fútbol, a partir de la identificación territorial y la distinción social, ya que los clubes "solo podían contar con copas o reconocimientos que, a la larga, no servían para compararlos con otros o, lo que es lo mismo: no tenían en su haber galardones con los cuales competir (presumir) frente a sus rivales" (Rodríguez, 2010, p. 53).

En Colombia, la consolidación de los equipos fue centralizada, primero, en ciudades principales, luego, en ciudades intermedias $y$, finalmente, en ciudades pequeñas, durante los siglos XX y XXI. De esta manera, es posible organizar los clubes ${ }^{3}$ en nacionales ${ }^{4}$, regionales ${ }^{5}$ y locales $^{6}$. La identidad futbolística se constituye a partir de la pertenencia territorial, la distinción social y, además, la historia deportiva de los clubes. Posteriormente, se agregan aspectos relacionados con "lo pasional, lo histórico, lo sociocultural, lo político-económico e incluso, lo étnico y lo sexual" (J. Castro, 20 I9, p. II I).Asimismo, los emblemas y los colores representados en la ropa deportiva o en las banderas son parte de la constitución de las identidades futbolísticas entre los simpatizantes, y en especial en los

3 En esta referencia, solo se consideran los clubes que participan en Liga y Torneo en 2019, campeonatos organizados por la División Mayor del Fútbol Colombiano (Dimayor). En Colombia se fundaron equipos que conservaron su participación en la primera o segunda categoría; no obstante, muchos desaparecieron.

4 América, Cali, Junior, Medellín, Millonarios, Nacional y Santa Fe.

5 Bucaramanga y Cúcuta; Once Caldas, Pereira y Quindío; Huila y Tolima; Real Cartagena y Unión Magdalena; Chicó y Patriotas.

6 Alianza,Atlético, Barranquilla, Bogotá, Cortuluá, Envigado, Equidad, Fortaleza, Jaguares, Leones, Llaneros, Orsomarso, Pasto, Real San Andrés, Rionegro, Tigres, Universitario y Valledupar. 
hinchas, pues los símbolos del club lo representan, lo identifican y lo particularizan.

La identificación de un hincha con su club lo lleva a asemejarse a unos y el lugar para manifestar el apoyo a un club es en el estadio; además, a través de las banderas es posible exponer la pertenencia a un territorio $y$, así, el hincha se distingue de otros y esas distinciones pueden generar conflictos entre ellos (Ferreyra, 200I). En los estadios, se producen y reproducen identificaciones simbólicas en los cantos y en las banderas de los hinchas, pues la identidad puede estar relacionada con lo territorial: nacimiento, residencia, barrial o departamental.Así, se constituyen las rivalidades en el fútbol y el conflicto entre los hinchas, pues son ellos quienes protegen y defienden la identidad futbolística, y promueven una división entre "amigos" y "enemigos", reconocidos con símbolos específicos (Cristiano, 2017). El proceso identitario tiene su fundamento en el antagonismo, pues el fútbol promueve una división del mundo en amigos y en enemigos; el partido es el momento adecuado para el enfrentamiento de los amigos contra los enemigos (Ferreiro, 2003).

La identidad está relacionada con el sentido de pertenencia, ya que los hinchas sienten su participación en el equipo y en la barra brava, por lo cual se constituye una unidad indivisible entre el hincha, el club y la barra, e igualmente en el grupo social construyen lazos de hermandad (Recasens, 1996). En el estadio, las torcidas organizadas apoyan a su equipo de fútbol para que logre una victoria, aunque si el club no triunfa los hinchas han cumplido su propósito y han desencadenado sus emociones. Esas emociones particularizan a la colectividad y le otorgan singularidad e identidad (Ferreira, 2008). De acuerdo con esto, en las barras bravas es posible la constitución de una nueva forma de identidad, ya que los hinchas integran distintos aspectos de sus vidas. Igualmente, la barra brava expone su identidad a través de los trapos (banderas) y los bombos (instrumento musical de percusión) que poseen los emblemas y los colores de su equipo, manifiestan su organización, su dedicación y su pasión, pues la posesión del bombo y de las banderas son muestra de la ubicación jerárquica (Parada, 2013).

Además, las canciones de los hinchas permiten la caracterización de la constitución de la identidad entre los $\mathrm{GOH}$. En los cantos de las hinchadas, se mezclan diversos géneros musicales y múltiples problemas ideológicos, no relacionados necesariamente con el fútbol (Gándara,
1999). Los cantos constituyen una representación identitaria de lo propio (el equipo y la hinchada) y lo contrario (el equipo y la hinchada rivales), y expresan amor, aliento y festejo e insulto, amenaza y violencia, respectivamente. En este sentido, los cantos exponen formas de construir una identidad activa frente a una pasividad de los otros, es decir, un "nosotros" que se enfrenta a un "otros", distinto y rival histórico, territorial y, así, deportivo. Los ejes están relacionados con la identidad, la diferenciación, la violencia y el machismo, expresados mediante el "aguante" (Burgos \& Brunet, 2000).

\section{De las barras bravas y las rivalidades del fútbol}

Al iniciar el FPC, los clubes no habían elaborado su historia deportiva, una tradición futbolística que se logra a partir de los logros obtenidos por los equipos y permite la configuración social de los simpatizantes y los hinchas. De esta forma, la constitución de los hinchas se genera entre las décadas de 1950 y de 1960. Después de esa configuración futbolística, se inicia (probablemente) la organización de las barras desde la década de 1970 hasta la década de 1980. Posteriormente, las barras bravas se organizaron en ciudades principales e intermedias de Colombia durante la década de 1990: Bogotá, Cali, Medellín, Barranquilla, Manizales, Bucaramanga, Neiva, Pereira e lbagué. Su organización transformó las maneras de alentar y las formas de distinguir al otro, al rival, como un enemigo constituido futbolísticamente en la competencia.

A principios de la década de 1990, en Bogotá se fundó Santa Fe de Bogotá (199I) de Club Independiente Santa Fe y Blue Rain (1993) de Club Deportivo Los Millonarios. En Cali, Furia Roja (199I) de América de Cali y Ultra Puteria Verde (1992) de Asociación Deportivo Cali. En Bogotá, se ubicaron en lateral norte Comandos Azules \# 13 (1996) de Millonarios y en lateral sur La Guardia Albi-Roja Sur (1997) de Santa $\mathrm{Fe}^{7}$. También, surgió Disturbio Rojo8 (1997) de Club América de Cali en Bogotá. En Cali, Frente Radical Verdiblanco (1996) de Cali y Barón Rojo Sur (1997) de América. En Medellín, Los Del Sur (1997) de Atlético Nacional y Rexixtenxia Norte (1998) de Deportivo Independiente Medellín. En Barran-

\footnotetext{
7 Las tribunas laterales se ubican detrás de los arcos o porterías del estadio Nemesio Camacho "El Campín”, posición que sigue el modelo argentino, uruguayo, paraguayo, chileno, peruano y ecuatoriano.

8 Hinchas de América de Cali, nacidos en Bogotá.
} 
quilla, Frente Rojiblanco Sur (1998) de Atlético Junior. En Manizales, Holocausto Norte (1998) de Once Caldas. En Bucaramanga, Fortaleza Leoparda Sur (1998) de Atlético Bucaramanga. En Neiva, Alta Tensión Sur (1998) de Atlético Huila. En Pereira, Lobo Sur (1999) de Deportivo Pereira.Y en Ibagué, Revolución Vinotinto Sur (2000) de Deportes Tolima.

En este aspecto, hinchar por un club constituye una identidad futbolística, establecida a partir de la pertenencia territorial, la distinción social y la historia deportiva. Sin embargo, la competencia produce las rivalidades entre los hinchas, que se fortalecieron a partir de factores pasionales, históricos, socioculturales, político-económicos e, incluso, étnicos y sexuales ajenos al fútbol; aumentadas con la formación de las barras bravas en Colombia. Las rivalidades futbolísticas son una construcción histórica que se definen a partir de la identidad de cada uno de los clubes de fútbol y, así, de sus hinchas, manifestada a través de representaciones contradictorias (constantemente resignificadas) en lo social, lo económico, lo político y lo cultural, constituyentes de ciertos significados y valores. Esas rivalidades son complementarias, pues el uno requiere de otro, $y$ viceversa. $Y$ en algunas ocasiones esas rivalidades desembocan en enfrentamientos (Levatti, 1998). En ese sentido, las prácticas sociales constituyen la identidad desde la experiencia social y cotidiana de los actores involucrados. $Y$ las rivalidades se construyen a partir de los siguientes factores: territorial, futbolístico, pasional y económico (Aragón, 20I I).

Un torcedor', al identificarse con un club de fútbol, se opone a los torcedores de otros equipos. Esa rivalidad es reflejo de algunos aspectos de la estructura social que simbolizan los equipos de fútbol desde su fundación, por ejemplo, la distinción entre la "élite" y el "pueblo", características que se reafirman en la ubicación territorial, aunque los torcedores no necesariamente pertenecen a esas "clases" (Louzada, 20I I). Desde otra perspectiva, los integrantes de las barras bravas constituyen su identidad a partir de acciones como no callarse, saltar, mantenerse en el lugar de la pelea y conservar los brazos en alto, es decir, incluyen en la barra brava a quienes participan activamente en las expresiones festivas y violentas, y excluyen a quienes no lo hacen. Igualmente, las diferencias identitarias o las rivalidades futbolísticas se manifiestan en aspec-

9 Hincha que forma una torcida organizada. tos territoriales, étnicos y culturales que se expresan en las canciones y en los enfrentamientos (J. Castro, 20I5).

Los aficionados se reconocen como incondicionales con el equipo y lo expresan en su animación y las rivalidades son un antagonismo en la competencia deportiva, expresión de las diferencias político-históricas, económico-sociales o culturales (Orellana, 20I2). Los equipos tienen ciertas características vinculadas con el origen, la historia y el estilo de juego, particularidades asumidas por los hinchas para representarse a sí mismos, e igualmente los símbolos y las prácticas sirven para reforzar los vínculos de pertenencia y distinguirse de otros seguidores (Aceves, 20I2). Las barras se constituyen a partir de compromisos y responsabilidades, prevalece la masculinidad y las rivalidades se manifiestan mediante la festividad y el "aguante" (Celestino, 20I2). La identidad, la representatividad y el orgullo de la población de un territorio son proyectadas en el equipo de fútbol y las rivalidades del fútbol son la expresión de distinciones históricas, étnicas, económicas, políticas y culturales (Macías, 20I2). La rivalidad histórica y geográfica de los equipos es admitida por los miembros de las barras, la cual constituye una identidad mediante la participación, la animación, el colorido y el folclor (Delgado \& González, 20I2).

La competencia deportiva de los clubes de fútbol desemboca en la constitución de las rivalidades de los hinchas y entre las barras bravas se consolidan mediante los insultos $y$, en especial, los enfrentamientos. En Colombia, antes de la década de 1990, es muy probable que los hinchas de los clubes nacionales y regionales protagonizaran peleas. Sin embargo, la cantidad de involucrados y la frecuencia de las riñas no fueron la portada o la noticia principal en radio, prensa o televisión, lo cual opacó su impacto o trascendencia. La formación de las barras bravas mostró una multitud de hinchas agrupados, quienes saltaban y cantaban en comunión y en armonía en las tribunas de los estadios. También se observó una muchedumbre de hinchas que agredían a grupos organizados semejantes, cuyos enfrentamientos fueron protagonizados principalmente por los hinchas que formaban Barón Rojo Sur, Comandos Azules \# 13, Disturbio Rojo Bogotá, Frente Radical Verdiblanco, Frente Rojiblanco Sur, La Guardia Albi-Roja Sur, Los Del Sur y Rexixtenxia Norte, barras bravas de los equipos en nacionales.

También los hinchas que formaban Alta Tensión Sur, Fortaleza Leoparda Sur, Holocausto Norte, Lobo Sur y Revolución 
Vinotinto Sur (barras bravas de los clubes regionales) protagonizaron riñas antes, durante o después de los encuentros futbolísticos, en interior o alrededor de los estadios. Igualmente, se constituyeron $\mathrm{GOH}$ de otros equipos regionales $y$, además, de los clubes locales, que también protagonizaron enfrentamientos. En otras palabras, los hinchas que forman las barras bravas de los clubes nacionales, regionales y locales se enfrentan sin distinción en distintos horarios y lugares. De esta manera, las peleas entre hinchas rivales que forman barras bravas de los clubes nacionales, regionales y locales lograron frecuencia y convocatoria.Asimismo, entre algunos hinchas en las barras bravas se emprendió la constitución de rivalidades internas, es decir, los rivales son hinchas del mismo equipo; dichas rivalidades provocaron divisiones internas que suscitaron la conformación de una nueva barra brava del mismo club de fútbol.

De Comandos Azules se originó (una nueva) Blue Rain, Frente Radical Verdi Blanco se fraccionó y el resultado fue Avalancha Verde Norte, de Frente Rojiblanco Sur se desprendió La Banda de los Kuervos y de Los Del Sur surgió Nación Verdolaga. Aunque la organización del Disturbio Rojo Bogotá y el Barón Rojo Sur fue independiente, en un principio mantenían una amistad, pero luego se distanciaron e iniciaron enfrentamientos. Las disputas entre Los Del Sur (Medellín) y Nación Verdolaga (Bogotá), así como entre Barón Rojo Sur (Cali) y Disturbio Rojo Bogotá muestran que, a pesar de las semejanzas futbolísticas, es decir, hinchar por el mismo club, no es suficiente, ya que las pertenencias territoriales producen los regionalismos, plasmados en los enfrentamientos. De acuerdo con esto, las rivalidades se pueden clasificar en endógenas (disputas entre hinchas del mismo equipo y con la Policía) y en exógenas (disputas con hinchas de distintos equipos) (González, 2012).

\section{Del "aguante" de las barras bravas}

En enero 5 de 1983, fue asesinado Raúl Servín Martínez, hincha de Boca Juniors ${ }^{10}$, por aficionados de Quilmes". Antes de disparar, los homicidas gritaron "aguante Quilmes", al parecer fue la primera vez que se utilizó oficialmente esta expresión. De esa manera, la categoría para comprender la violencia en el fútbol es el "aguan-

10 Club Atlético Boca Juniors, institución deportiva de la Ciudad Autónoma de Buenos Aires.

II Quilmes Atlético Club, institución deportiva de la provincia de Buenos Aires. te", pues es una masculinidad, exponente de marcas y cicatrices en el cuerpo, y es un código de honor que, al ser vulnerado, debe ser vengado $y$, además, es necesario demostrarlo (individual y colectivamente) frente al otro, mediante la violencia (Alabarces, 20I2). Desde esa perspectiva, el "aguante" "exige estar dispuesto constantemente a la pelea, comprobando en la agresión física aquello que es la última y más valedera verificación: la capacidad de resistir y metabolizar los golpes" (Elbaum, 1998, p. 159).

Los miembros de las barras bravas se involucran en enfrentamientos como resultado del encuentro en zonas aledañas al estadio o en los barrios de distintas ciudades. El enfrentamiento - una lucha cuerpo a cuerpo y en los casos más peligrosos pueden utilizar armas blancas o de fuego- busca agredir al adversario, quien en algunas ocasiones puede resultar herido e, incluso, ser asesinado. De acuerdo con lo señalado, Gordo Lan ${ }^{12}$ sostiene:

En una pelea se ve el "aguante", por lo menos en una pelea se ve mucho. Se desgasta uno terrible, pues el "aguante" es resistir. Así a usted le den roca $^{13}$, palo' ${ }^{14}$, usted ahí con el "aguante", está parado' en la suya y no da, no da un paso a atrás, [eso] es tener "aguante".

La pelea con el rival, tener "aguante", es pararme en la calle solo, ver a cinco y decir: Bueno gonorrea ${ }^{16}$, me voy a montar de un palo y sabe que me voy a dar con los cinco. Tengo "aguante", me le banco ${ }^{17}$ lo que sea, [eso] es tener "aguante".

Los integrantes de las barras bravas que asumen los enfrentamientos lo hacen porque son capaces de recibir y tolerar los golpes, pues saben que pueden defenderse y también proporcionarlos. El enfrentamiento es un comportamiento regular entre los $\mathrm{GOH}$, ya que posibilita el respeto y propone un modelo a seguir, pues "lo particular es que la lucha por el reconocimiento se basa en un paradigma de masculinidad agresiva, cosificado en la figura del guerrero callejero" (Panfichi, 1999, p. 160). Las peleas o las riñas en las que se involucran los integrantes de las barras

12 Actualmente es uno de los referentes de Comandos Azules de Millonarios.

13 Piedras.

14 Palo de madera.

15 Se mantiene en el lugar de la pelea.

16 En este contexto, se refiere a tomar la decisión de afrontar el enfrentamiento, recurriendo a un lenguaje soez.

17 Soportar. 
bravas tienen como consecuencia las huellas en el cuerpo, puesto que intervenir implica recibir lo mismo que se ha dado. La participación le otorga al hincha ciertos beneficios que solo tienen validez e importancia en el grupo. "Por el sacrificio, por las medallas, (cicatrices y capturas policiales) se purificarán, hacia dentro de ellos mismos y hacia el juicio de sus pares, porque ganan admiración, respeto, y escalan posiciones en la jerarquía del grupo" (R. Castro, 1999, p. 218).

En otras palabras, propinar golpes y recibirlos o terminar retenido por la Policía le concede al integrante de la barra brava un ascenso en la posición en el grupo, ya que sale del anonimato y adquiere el respeto de sus semejantes, pues se involucra seriamente en todas las actividades de la barra. Además, le concede ciertos beneficios, que son importantes en la agrupación, privilegios que muchos aspiran. El "aguante" posee un valor que es estimado en la barra.

La ideología del aguante representa un principio aglutinador de la experiencia, definido como el arte de no escapar, de soportar lo que venga. La ideología del aguante alteriza el ideal de masculinidad del hincha e implica el despliegue de un sentimiento estoico ante la adversidad. En este sentido se extrema la identificación de la barra con la ideología del aguante toda vez que se afirma que un hincha sin aguante no es un hincha.

La ideología del aguante somete continuamente al hincha a su confrontación posibilitando su autoafirmación. El aguante no se puede "soltar" o "aflojar", siempre se debe estar allí, ofreciendo "todo". En este sentido el aguante implica un ejercicio de sacrificio por parte del hincha, sacrificio no exento de dolor, que pone a prueba la resistencia masculina otorgándole un plus de honor. (Abarca, 200 I, p. II5)

En otros términos, "el aguante supone un ideal masculino marcado por la agresividad, el dinamismo, los actos valientes, el compañerismo y no rendirse ante el peligro" (Gil, 2007, p. 42). Por tanto, el "aguante" es una masculinidad agresiva que muestra la fuerza del hincha ante las adversidades. De esa manera, le es concedido el respeto y la reputación frente a los demás integrantes de la barra. Por ese motivo, el "aguante" requiere, por lo menos, dos actores. Entonces, ¿quién tiene más "aguante"? Cada barra se autoproclama portadora del "aguante", es decir, festiva y combatiente. $Y$ censura a la barra contraria por ser, aparentemente, amarga y, además, cobarde. Los integrantes de los $\mathrm{GOH}$ reconocen lo que llevan a cabo desde la gradería y enaltecen la presencia que marcan en las calles. Mientras que a los adversarios los descalifican en el enfrentamiento simbólico y buscan ridiculizarlos en el "combate" callejero, pues es en esa acción que ratifican lo que han señalado.

La barra brava que canta pero no pelea no es una barra que posea "aguante", y viceversa, ya que el "aguante" es un patrimonio que se ha ganado rompiendo la voz en la tribuna, saltando sin parar, manteniendo los puños enfrente y no retrocediendo en el enfrentamiento contra el rival. De ese modo, el "aguante" se constituye en un estilo de vida y en un modelo de las nuevas generaciones.

Los integrantes de las hinchadas no pierden oportunidad para señalar que el "aguante" es para ellos un bien que se disputa en los conflictos violentos. El recuerdo de prácticas donde probaron tener "aguante" es parte constitutiva de sus discursos y sus canciones. Hacer visibles estas acciones, mostrarse como participantes de acciones violentas, es para los hinchas exhibir la posesión del "capital aguante". Este capital es fuente de orgullo y estatus, por tal motivo debe exhibirse. (Garriga \& Moreira, 2006, p. 7I)

El "aguante" no es para ocultarlo, pues es necesario demostrárselo al otro -al semejante y al diferente-, ya que se exhibe como una huella que es capaz de hacer frente a las adversidades, sobre todo, si los rivales superan significativamente en número, es decir, si se lleva la desventaja porque permite demostrar una capacidad para aguantar los reveses. El "aguante" ordena la jerarquía de la barra y ubica a aquellos que han sido capaces de demostrarlo -en especial, a través del enfrentamientoen los puestos más altos, y esa posición les permite obtener entradas a los partidos, ingreso al estadio con horas de anterioridad, facilidades en los viajes, ropa oficial del equipo, cercanía con los jugadores y los directivos, entre otras ventajas.

Los comportamientos en los que se hace evidente el conflicto, la agresividad y la violencia o, simplemente, el "aguante", difícilmente pueden desaparecer del entorno de las barras bravas, pues otorga respeto, admiración e influencia en el grupo y, además, patrocinios. El "aguante" se orienta a una actuación violenta que mantiene el conflicto, ya que conserva la particularidad como seguidor del equipo e integrante de la barra, y prolonga la diferencia con los rivales. "El aguante tiene una clara connotación violenta y esta violencia no puede ser erradicada sin per- 
der la identidad como hincha, tanto en el discurso, como en las formas físicas en que se manifiesta y se representa" (Aragón, 2007, p. 75).

El "aguante" puede ser entendido por aquellos que no hacen parte de las barras bravas como conductas violentas en los momentos en los cuales se dan los enfrentamientos entre los hinchas (rivales o del mismo equipo) y la intervención de la Policía para contenerlos. En otras palabras, el "aguante" visibiliza la agresividad, el conflicto y la violencia entre las barras bravas. Sin embargo, los integrantes de las barras bravas le han otorgado un sentido diferente, pues está relacionado con la valentía, la hombría, el orgullo o el honor. Dicho de otro modo, el "aguante" funciona como un elemento de cohesión, de homogeneización y de distinción en los $\mathrm{GOH}$, que lleva a asumir la pertenencia al grupo y al equipo. El "aguante" es una noción-experiencia particular de las barras bravas de Hispanoamérica.

\section{"Desde el cielo te voy a alentar"18}

Entre 2004 y 2018 , en Colombia, alrededor de 154 personas han sido asesinadas en ataques o riñas que involucraron a hinchas que forman barras bravas ${ }^{19}$. Por estos crímenes han sido condenadas cerca de 23 personas. Sin embargo, es necesario señalar que no es posible consultar cifras oficiales, ya que el Instituto Nacional de Medicina Legal y Ciencias Forenses no particulariza el homicidio, es decir, si fue asesinado por pertenecer a una barra brava o por participar en enfrentamientos entre hinchas, aunque en el informe Forensis 2014 datos para la vida: Herramienta para la interpretación, intervención y prevención de lesiones de causa externa en Colombia se afirma que una de las causas que son generadoras de la violencia en Colombia es la pertenencia a las barras de fútbol (20|4, p. I33). De esta manera, las cifras presentadas fueron recopiladas a partir de noticias periódicas de El Colombiano, El Espectador, El Heraldo, El País, El Tiempo y Semana ${ }^{20}$, usando los descriptores "hinchas", “asesinados", "barras bravas", “Colombia”.

18 El título y el subtítulo del artículo provienen de una canción de Comandos Azules de Millonarios: "Gloria mi vida gloria mi pasión, Millonarios querido de mi corazón. El día que me muera me vas a escuchar, desde el cielo te voy a alentar".

19 La cifra de hinchas asesinados no incluye los homicidios de Juan Manuel Bermúdez Nieto y Alex Julián Gómez Ramírez (hinchas que formaban Disturbio Rojo), quienes fueron ultimados el 19 de mayo de 2002 por paramilitares en la vía Medellín-Puerto Berrío. Asimismo, no se consideran los hinchas que han fallecido por sobredosis, en accidentes, crímenes pasionales o por su participación en bandas delincuenciales.

20 Por ese motivo, los números pueden variar.
Por otra parte, en 2004 fue registrado, por distintos medios de información, el primer homicidio en el que estuvieron involucrados integrantes de barras bravas. Sin embargo, no es posible asegurar que no hubo homicidios antes de ese suceso (Figura I).

Este número de homicidios supone un panorama de violencia ligada al fútbol de Colombia.Aunque si se comparan las cifras con los asesinatos provocados por miembros de la fuerza pública, la guerrilla, los paramilitares-bandas criminales, los traficantes de drogas o la delincuencia común, entre otros victimarios, los números de los muertos relacionadas con la violencia del fútbol son mínimos. Por ejemplo, entre 2004 y 2018 , fueron asesinados $226299^{21}$ ciudadanos colombianos en territorio nacional, es decir, los hinchas asesinados representan el 0,068 \%. Lo que llama la atención de las cifras de fallecidos es el supuesto que alrededor del fútbol la violencia debería reducirse e, incluso, desaparecer, pues el deporte podría contribuir a su disminución. Sin embargo, al parecer no lo ha logrado; por el contrario, la ha mantenido y la ha fortalecido.

No obstante, las cifras de asesinados relacionados con la violencia del fútbol en Colombia es bastante elevada respecto de otros países. En Argentina, entre 1922 y 2018 (96 años), fallecieron 332 personas relacionadas con el fútbol. Las causas de las muertes son múltiples: golpes, peleas, pedradas, acuchillados, disparos, accidentes, entre otros $^{22}$. Dicho de otra manera, Argentina tiene un promedio de 3,46 fallecidos por año. Sin embargo, en Colombia, entre 2004 y 2018 (14 años), fueron asesinadas I54 personas, es decir, registra un promedio de II crímenes por año. En este aspecto, Colombia registra 3,18 veces más muertes por la violencia del fútbol que Argentina. En Colombia, los crímenes han involucrado a hinchas que han asesinado a seguidores rivales; a fanáticos del mismo equipo, a personas ajenas a la afición futbolística e, incluso, en el ejercicio de miembros de la Policía Nacional, quienes ejercen mayor violencia para reducir a los violentos del fútbol (Figura 2).

En este aspecto, los crímenes se han efectuado en las graderías de los estadios y en sus alrededores; en barrios,

2I La cifra resultante fue tomada de los informes intitulados Forensis: datos para la vida del Instituto Nacional de Medicina Legal y Ciencias Forenses, en el periodo de 2004 a 2018.

22 La cifra fue tomada de la organización no gubernamental Salvemos al Fútbol (s. f.), que tiene como propósito luchar contra la violencia y la corrupción en el fútbol de Argentina. 
Figura I. Asesinatos por años entre 2004 y 2018.

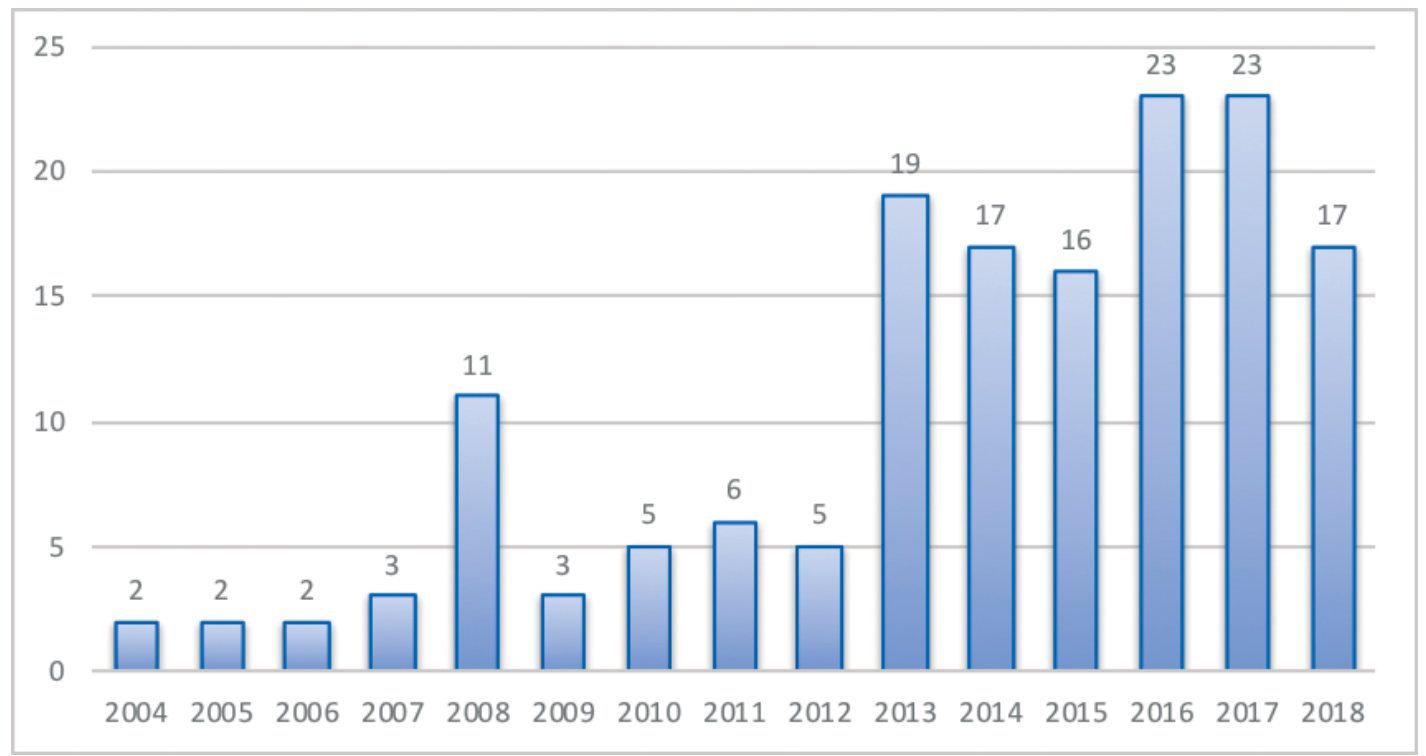

Figura 2. Distribución de los hinchas asesinados.

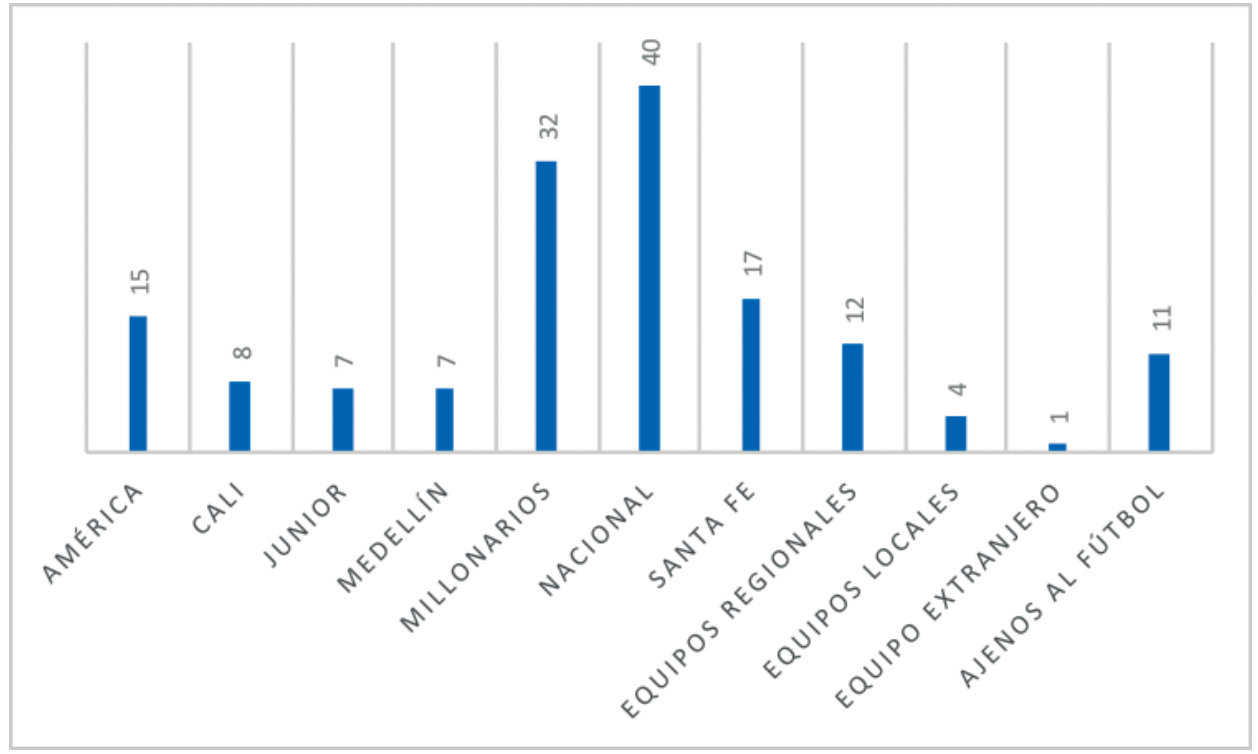

vías principales o parques de ciudades; en carreteras mientras viajan los hinchas entre las ciudades; e, incluso, en territorio extranjero. En otras palabras, los crímenes en su mayoría se han cometido en sitios lejanos de los estadios, en horarios distintos de los encuentros futbolísticos y en lugares diferentes del origen territorial de los clubes. No obstante, en el balance presentado, no se considera una cifra indeterminada de hinchas que sufrieron graves lesiones, otros que quedaron inválidos o perdieron alguna extremidad. Por ejemplo, un hincha de Millonarios fue gravemente agredido con machete por hinchas de Unión Magdalena, un hincha de Nacional perdió sus extremidades superiores en un ataque con machete efec- tuado por hinchas de Bucaramanga; un hincha de Santa Fe después de una brutal agresión por parte de hinchas de Millonarios perdió parte de su cráneo; y un alto número de fanáticos que quedaron en sillas de ruedas después de participar en riñas entre hinchas.

En este sentido, desde 1999, se han promovido campañas y se ha propuesto una política pública contra la violencia del fútbol. El 5 de septiembre de 1999, inició la campaña "Jugando limpio todos ganamos" del programa Misión Bogotá. Esta campaña fue presentada antes de iniciarse el clásico capitalino y tuvo como objetivo crear espacios de tolerancia en el fútbol. Su iniciativa promovió que los 
líderes de las barras bravas —Comandos Azules \# 13 de Millonarios y La Guardia Albi-Roja Sur de Santa Fecontuvieran los comportamientos agresivos entre los hinchas. Además, es propuesto el Decreto 523/1999, de 12 de agosto, por el cual se reglamenta la realización de eventos deportivos en el Estadio Nemesio Camacho El Campín y otros escenarios deportivos del Distrito Capital, con ocasión del campeonato de fútbol profesional. Posteriormente, se establece el Decreto 164/2004, de 31 de mayo, por el cual se crea el comité de seguridad y convivencia para los espectáculos de fútbol profesional "Goles en Paz". Luego, se implementa el Decreto $455 / 2008$, de 23 de diciembre, por el cual se reglamenta la realización de los partidos de fútbol de carácter profesional en el Estadio Nemesio Camacho El Campín y otros escenarios deportivos del Distrito Capital y se deroga el Decreto 523 de 1999, el cual regulaba la materia".

Sin embargo, las medidas tomadas por las autoridades distritales no han evitado que los integrantes de las barras bravas participen en comportamientos agresivos que pueden desembocar en el vandalismo, en los enfrentamientos y en los asesinatos. En consecuencia, la política pública se hace relevante y necesaria, para atender un problema que atenta contra el desarrollo de los encuentros futbolísticos y la convivencia en los estadios. En este aspecto, la violencia del fútbol se ha incluido en la agenda nacional de políticas públicas. Como resultado, se han aprobado la Ley 1270/2009, de 5 de enero, por la cual se crea la Comisión Nacional para la Seguridad, Comodidad y Convivencia en el Fútbol y se dictan otras disposiciones, y el Decreto 1007/2012, de 16 de mayo, por el cual se expide el Estatuto del Aficionado al Fútbol en Colombia.Además, otras que vinculan la violencia en eventos deportivos: Ley 1445/20 I I, de 12 de mayo, por medio de la cual se modifica la Ley $18 \mathrm{I}$ de 1995, las disposiciones que resulten contrarias y se dictan otras disposiciones en relación con el deporte profesional; la Ley I453/20 I I, de 24 de junio, por medio de la cual se reforma el Código Penal, el Código de Procedimiento Penal, el Código de Infancia y Adolescencia, las reglas sobre extinción de dominio y se dictan otras disposiciones en materia de seguridad; y la Ley I80I/20I6, de 29 de julio, por la cual se expide el Código Nacional de Seguridad y Convivencia Ciudadana.

Pero la Ley 1270/2009, de 5 de enero, propone estrategias de seguridad y convivencia en los espectáculos deportivos como ya habían sido planteadas en el Decreto 523/1999, de 12 de agosto, el Decreto 164/2004, de 31 de mayo y el Decreto 455/2008, de 23 de diciembre. En otras palabras, se plantea lo que ya se ha propuesto. Aunque el propósito de las normas establecidas es reducir los índices de violencia del fútbol, es decir, disminuir los enfrentamientos entre hinchas que han provocado el asesinato de un centenar de ciudadanos en Colombia y, además, el daño a la propiedad pública y privada, los decretos y las leyes establecidas proponen, una y otra vez, una serie de medidas y de sanciones que no han logrado la trascendencia esperada. En otras palabras, el panorama de violencia en el contexto del fútbol en Colombia está lejos de desaparecer o reducirse, pues los comportamientos agresivos y conflictivos de los hinchas son reconocidos positivamente entre los hinchas en las barras bravas, puesto que son la manifestación del "aguante". Además, la implementación de las normas no implica su inmediato cumplimiento.

A pesar de la violencia del fútbol en Colombia,Villanueva y Rodríguez (2013) recalcan los aspectos positivos de la legislación instituida al prevenir los enfrentamientos entre las barras futboleras y estandarizar las acciones que permitan la seguridad, la evacuación, el transporte y el desplazamiento de las hinchadas. En este aspecto,Amaya (2016) destaca la necesidad de acatar lo estipulado en la legislación colombiana relacionada con las "barras futboleras" y de alfabetizar a los hinchas e involucrarlos en la elaboración de una política pública que incluya aspectos de convivencia, seguridad y comodidad. Dicho de otra manera, es probable que la violencia del fútbol fuese más compleja o más grave si la política pública no se hubiese instaurado; quizá, habría más violencia de la violencia existente.

\section{Conclusiones}

Los clubes de fútbol constituyen identidades deportivas y la competencia produce las rivalidades, ya que pone frente a frente a dos contendientes en el terreno de juego. Sin embargo, las barras bravas llevan al extremo las rivalidades y son manifestadas a través del "aguante", una noción-experiencia que representa un valor positivo entre los hinchas que forman las barras bravas y se realiza en dos escenarios: en la tribuna $y$, en especial, en las calles, es decir, el "aguante" es una noción y una experiencia que se entiende y vive en las barras bravas. El "aguante" en la tribuna expresa comportamientos festivos y en la calle son conductas violentas, es decir, el "carnaval" y el "combate". Este artículo hizo énfasis en el "aguante" como "combate", es decir, como violencia. Desde esa perspectiva, el "aguante" es una norma callejera que busca la supremacía del más fuerte y funciona solo en el enfrentamiento, 
ya que las palabras no sirven para nada, "se las lleva el viento", y simplemente queda afrontar la pelea.

En este sentido, en los enfrentamientos (entre los hinchas que forman las barras bravas), se ha normalizado el uso de armas (blancas o de fuego), sin importar, al parecer, las consecuencias de su uso, pues ha dejado una cifra elevada de ciudadanos asesinados en comparación con otros países, pero minúscula respecto de otras violencias en Colombia. Lo que llama la atención de este tipo de acciones agresivas, conflictivas y violentas es que pueden producir una respuesta similar a la causada y generar un círculo del que no es fácil salir. La violencia en el contexto del fútbol no es espontánea, ni accidental, puesto que son la consecuencia del extremismo de las rivalidades deportivas protagonizada por los hinchas que forman las barras bravas y se agrava la problemática, debido a que son la demostración de la valentía, la hombría, el orgullo o el honor o el "aguante".

El "aguante" es una noción-experiencia contradictoria con la seguridad ciudadana. Por eso, se ha propuesto una serie de decretos y leyes que pretenden reducir los índices de violencia ligada al fútbol. No obstante, las medidas establecidas proponen una y otra vez un conjunto de medidas y de sanciones que no han logrado la trascendencia esperada. El programa Goles en Paz y la Comisión Nacional para la Seguridad, Comodidad y Convivencia en el Fútbol tampoco han arrojado los resultados proyectados. Por tanto, la implementación de las normas no implica su inmediato cumplimiento, es decir, los índices de violencia no se han reducido y el comportamiento violento de las barras bravas tampoco se ha modificado, a pesar de la legislación existente. Aunque aquellos han mostrado la intención de prevenir las conductas violentas de los hinchas, el "aguante" tiene mayor legitimidad entre los integrantes de las barras bravas.

\section{Referencias}

Abarca, H. (200I). Crónicas del aguante. En J. Olavarría (Ed.), Hombres: identidad/es y violencia. (pp. I I I-124). Santiago de Chile, Chile: Facultad Latinoamericana de Ciencias Sociales. Recuperado de http://joseolavarria.cl/wp-content/ uploads/downloads/2010/10/2do-Encuentro-HombresIdentidad-y-violencia.pdf

Aceves, R. (20I2). Aficionados futboleros en Guadalajara: características y representaciones de rojiblancos, rojinegros y tecolotes. En R. Magazine, S. Martínez, \& S. Varela (Coords.), Afición futbolística y rivalidades en el México contemporáneo: una mirada nacional. (pp. 89-1 I4). Ciudad de México, México: Universidad Iberoamericana.
Alabarces, P. (2012). Crónicas del aguante: fútbol violencia y politica. Buenos Aires, Argentina: Capital Intelectual.

Amaya, A. (2016). Leyes, realidades y desafíos. En R. Soto Lagos, \& O. Fernández Vergara (Comps.), ¿Quién raya la cancha? Visiones, tensiones y nuevas perspectivas en los estudios socioculturales del deporte en Latinoamérica. (pp. 177-190). Buenos Aires, Argentina: Consejo Latinoamericano de Ciencias Sociales.

Aragón, S. (2007). “Los trapos se ganan en combate”: una mirada etnográfica sobre las representaciones y prácticas violentas de la "barra brava" de San Lorenzo de Almagro. Lanús, Argentina:Antropofagia.

Aragón, S. (20II). La construcción de identidades y rivalidades futbolisticas en Buenos Aires. Esporte e Sociedade, 6(17). Recuperado de http://www.uff.br/esportesociedade/pdf/ es I 702.pdf

Benninghoff, F. (200I). “Cuánta tierra civilizada hay en Colombia?”: guerras, fútbol y élites en Bogotá 1850-1920 (Tesis de grado). Universidad Nacional de Colombia, Bogotá, Colombia).

Burgos, R., \& Brunet, M. (2000). Un análisis de los cantos de los hinchas de Gimnasia y Esgrima de Jujuy. Lecturas, Educación Física y Deportes, I (26), I-4. http://www.efdeportes.com/efd26a/jujuy.htm

Cárdenas,W. (20I5). Forensis 20 I 4: Datos para la vida. Bogotá: Instituto Nacional de Medicina Legal y Ciencias Forenses.

Castro, J. (2015). Identidad y rivalidad en una barra brava en Bogotá. Revista de Antropología y Sociología: Virajes, I7(I), 207-228.

Castro Lozano, J. A. (2019). De las rivalidades a la violencia del fútbol en Colombia. Revista Brasileira de Ciências do Esporte, 4/(I), 109-II5. http://dx.doi.org//0.1016/j. rbce.2018.07.006

Castro, R. (1999). Un día de partido. Comunidades sentimentales y rituales violentos en la Trinchera Norte. En A. Panfichi, \& M. Valcárcel (Eds.), Juventud: sociedad y cultura. (pp. 173222). Lima, Perú: Pontificia Universidad Católica del Perú.

Celestino, M. (2012). El clásico norteño Monterrey vs. Tigres: una 'rivalidad' mediática. En R. Magazine, S. Martínez, \& $\mathrm{S}$. Varela (Coords.), Afición futbolística y rivalidades en el México contemporáneo: una mirada nacional. (pp. I|5-|4|). Ciudad de México, México: Universidad Iberoamericana.

Cristiano, J. (2017). La música de las tribunas. Identidad y violencia en los cantos de los hinchas de fútbol y básquetbol. En R. Bayce García Lagos, \& B. Mora Pereyra (Coords.), Violencia en el deporte: discursos, debates y políticas en Uruguay. (pp. 123-139). Montevideo, Uruguay: Universidad de la República. 
Decreto 523/1999, de 12 de agosto, Por el cual se reglamenta la realización de eventos deportivos en el Estadio Nemesio Camacho El Campín y otros escenarios deportivos del Distrito Capital, con ocasión del campeonato de fútbol profesional. Registro Distrital, núm. 1960 (1999).

Decreto 164/2004, de 31 de mayo, por el cual se crea el comité de seguridad y convivencia para los espectáculos de fútbol profesional "Goles en Paz". Registro Distrital, núm. 3108 (2004).

Decreto 455/2008, de 23 de diciembre, por el cual se reglamenta la realización de los partidos de fútbol de carácter profesional en el Estadio Nemesio Camacho El Campín y otros escenarios deportivos del Distrito Capital y se deroga el Decreto 523 de 1999, el cual regulaba la materia". Registro Distrital, núm. 4I 24 (2008).

Decreto 1007/2012, de 16 de mayo, por el cual se expide el Estatuto del Aficionado al Fútbol en Colombia. Diario Oficial, núm. 48432 (20I2).

Delgado, E., \& González, J. M. (2012). De "Los de arriba" a "Los hijos de la mermelada": barrismo y música en El Bajío. En R. Magazine, S. Martínez, \& S. Varela (Coords.), Afición futbolistica y rivalidades en el México contemporáneo: una mirada nacional. (pp. 153-174). Ciudad de México, México: Universidad lberoamericana.

Elbaum, J. (1998). Apuntes para el 'aguante'. La construcción simbólica del cuerpo popular. En P.Alabarces, R. Di Giano, \& J. Frydenberg (Comps.), Deporte y sociedad. (pp. 157162). Buenos Aires, Argentina: Editorial Universitaria de Buenos Aires.

Ferreira, M. (2008). A torcida rubro-negra no Maracanã. Esporte e Sociedade, 3(8). Recuperado de http://www.uff.br/ esportesociedade/pdf/es807.pdf

Ferreiro, J. P. (2003). 'Ni la muerte nos va a separar, desde el cielo te voy a alentar': apuntes sobre identidad y fútbol en Jujuy. En P. Alabarces (Comp.), Futbologías: fútbol, identidad y violencia en América Latina. (pp. 57-69). Buenos Aires, Argentina: Consejo Latinoamericano de Ciencias Sociales. Recuperado de https://www.clacso.org.ar/librerialatinoamericana/buscar_libro_detalle.php?id_libro $=237 \&$ campo $=$ titulo\&texto $=$ futbol

Ferreyra, A. (200I). Lugares, hombres y banderas de fútbol. Temas y problemas de comunicación, II, 8I-90.

Gándara, L. M. (1997). Las voces del fútbol: análisis del discurso y cantos de cancha. Literatura y Lingüística, 10, 43-66.

Garriga Zucal,J., \& Moreira, M.V. (2006). “El aguante: hinchadas de fútbol, entre la pasión y la violencia. En D. Míguez, \& P.
Semán (Eds.), Entre santos, cumbias y piquetes: las culturas populares en la Argentina reciente. (pp. 55-73). Buenos Aires, Argentina: Biblos.

Gil, G. J. (2007). Hinchas en tránsito: violencia, memoria e identidad en una hinchada de un club del interior. Mar del Plata, Argentina: Universidad de Mar del Plata.

González Ponde de León, M. Á. (20I2). Rivalidades sociales y culturales en torno a un grupo de animación de un equipo de fútbol: el caso de la Ultra I90I de Pachuca. En R. Magazine, S. Martínez, \& S.Varela (Coords.) (Coords.), Afición futbolistica y rivalidades en el México contemporáneo: una mirada nacional. (pp. 287-3।3). Ciudad de México, México: Universidad Iberoamericana.

Instituto Nacional de Medicina Legal y Ciencias Forenses. (2019). Forensis 2018 datos para la vida: Herramienta para la interpretación, intervención y prevención de lesiones de causa externa en Colombia. Bogotá, Colombia: Autor. Recuerado de http://www.medicinalegal.gov.co/cifras-estadisticas/forensis

Jaramillo Racines, R. (20I8). Ensayos sociológicos sobre el fútbol en Colombia. En R. Jaramillo Racines, G. E. Gómez, \& J.A. Castro, Fútbol y barras bravas: análisis de un fenómeno urbano. (pp. 23-225). Bogotá, Colombia: Siglo del Hombre.

Ley 1270/2009, de 5 de enero, por la cual se crea la Comisión Nacional para la Seguridad, Comodidad y Convivencia en el Fútbol y se dictan otras disposiciones. Diario Oficial, núm. 47223 (2009).

Ley I445/20 I I, de 12 de mayo, por medio de la cual se modifica la Ley $18 \mathrm{I}$ de 1995, las disposiciones que resulten contrarias y se dictan otras disposiciones en relación con el deporte profesional. Diario Oficial, núm. 48067 (20II).

Ley $1453 / 20$ II, de 24 de junio, por medio de la cual se reforma el Código Penal, el Código de Procedimiento Penal, el Código de Infancia y Adolescencia, las reglas sobre extinción de dominio y se dictan otras disposiciones en materia de seguridad. Diario Oficial, núm. 48I I0 (20II).

Ley I80 I/2016, de 29 de julio, por la cual se expide el Código Nacional de Seguridad y Convivencia Ciudadana. Diario Oficial, núm. 49949 (2016).

Levatti, A. (1998). Colón-Unión: violencia y rivalidad. En P. Alabarces, R. Di Giano, \& J. Fridenberg (Eds.), Deporte y sociedad. (pp. 103-109). Buenos Aires, Argentina: Editorial Universitaria de Buenos Aires.

Louzada, R. (2016). Identidade e rivalidade entre os torcedores de futebol da cidade de São Paulo. Esporte e Sociedade, 6. Recuperado de https://www.ludopedio.com.br/v2/ content/uploads/I04406_es I 708.pdf 
Macías Cervantes, C. F. (2012). ¿El clásico del Bajío? Algunas consideraciones históricas sobre una rivalidad social. En R. Magazine, S. Martínez, \& S. Varela (Coords.) (Coords.), Afición futbolística y rivalidades en el México contemporáneo: una mirada nacional. (pp. I43-152). Ciudad de México, México: Universidad Iberoamericana.

Orellana Suárez, J. G. (20I2). Los Pumas de la UNAM y sus rivalidades. En R. Magazine, S. Martínez, \& S. Varela (Coords.) (Coords.), Afición futbolística y rivalidades en el México contemporáneo: una mirada nacional. (pp. 61-85). Ciudad de México, México: Universidad Iberoamericana.

Panfichi,A. (1999). Representación y violencia en el fútbol peruano: barras bravas. Contratexto, 12, 15I-16I. Recuperado de http://revistas.ulima.edu.pe/index.php/contratexto/article/download/726/698

Parada Dueñas, F. J. (20|3). Barras bravas tensiones y convergencias desde una perspectiva híbrida. Revista de Ciencias Sociales, 3 I, 6I-85.

Recasens Salvo, A. (1996). Diagnóstico antropológico de las barras bravas y de la violencia ligada al fútbol. Santiago de Chile, Chile: Universidad de Chile.
Rodríguez Melendro, N. F. (20I0). Fútbol y afición. Proceso de las figuraciones en la manera de alentar a los equipos profesionales capitalinos (Santa Fe y Millonarios): la época de "El Dorado"y los años 80-90 (Tesis de maestría, Universidad Nacional de Colombia, Bogotá, Colombia). Recuperado de http://www.bdigital.unal.edu.co/2655/

Salvemos al Fútbol. (s. f.). Muertes en la historia por la violencia en el fútbol argentino. Recuperado de http://salvemosalfutbol.org/lista-de-victimas-de-incidentes-de-violencia-enel-futbol/

Santos Molano, E. (2005). Fútbol: una pasión incontenible. Revista Credencial Historia, 185. Recuperado de http://www. revistacredencial.com/credencial/historia/temas/futboluna-pasion-incontenible

Villanueva, A., \& Rodríguez Melendro, N. F. (20I3). Aspectos legales, jurídicos y normativos sobre barras futboleras en Bogotá y Colombia. En J. Garriga Zucal (Comps.), Violencia en el fútbol: investigaciones sociales y fracasos políticos. (pp. 253-264). Buenos Aires, Argentina: Ediciones Godot. 